\title{
Modelos de crescimento animal para tempos irregulares
}

\author{
Fernando Ribeiro Cassiano(1) e Thelma Sáfadi(1)
}

(1)Universidade Federal de Lavras, Departamento de Ciências Exatas, Campus Universitário, Caixa Postal 3037, CEP 37200-000 Lavras, MG, Brasil. E-mail: fernandorc19@yahoo.com.br, safadi@dex.ufla.br

Resumo - O objetivo deste trabalho foi propor modelos que considerem estrutura irregular dos dados e avaliá-los em relação a modelos utilizados com tempos regulares. Foram considerados os modelos de crescimento Gompertz, Logístico e Von Bertalanffy com estruturas regular e irregular para os erros. A metodologia foi exemplificada com o uso de dados reais e simulados. Foram utilizados 16 pesos médios de 160 animais da raça Hereford, com pesagens do nascimento até aproximadamente 2 anos de idade. Para cada modelo, os parâmetros do melhor ajuste foram utilizados para simulação. $\mathrm{O}$ ajuste dos modelos melhora quando a estrutura original dos dados é levada em consideração, com redução na soma de quadrados dos resíduos e no valor do critério de Akaike.

Termos para indexação: autocorrelação, ganho de peso, Hereford, modelos não lineares.

\section{Animal growth models for irregular times}

\begin{abstract}
The objective of this work was to propose models that consider an irregular data structure and to evaluate them in relation to models used for regular times. The Gompertz, Logistic, and Von Bertalanffy growth models, with regular and irregular structures for the errors, were considered. The methodology was exemplified using real and simulated data. Sixteen average weights of 160 animals of the Hereford breed were used, with weighing performed from birth up to approximately 2 years of age. For each model, the parameters of the best adjustment were used for the simulation. The model adjustments improve when the original structure of the data is considered, reducing the sum of the squares of the residues and reducing the value of the Akaike criterion.
\end{abstract}

Index terms: autocorrelation, weight gain, Hereford, nonlinear models.

\section{Introdução}

A busca pelo conhecimento do padrão de crescimento animal, seja ele populacional ou individual, tem motivado a proposição de diversos modelos matemáticos. Do ponto de vista prático, esse conhecimento permite que sejam adotadas estratégias para potencializar ou amenizar determinadas características do objeto de estudo. Geralmente, os modelos matemáticos utilizados em trabalhos sobre crescimento animal consistem de funções não lineares, com número pequeno de parâmetros.

Diversos trabalhos utilizaram modelos não lineares (Mazzini et al., 2005; Costa et al., 2009; Gomiero et al., 2009; McManus et al., 2010; Souza et al., 2010; Gonçalves et al., 2011; Mazucheli et al., 2011; Silva et al., 2011; Mansano et al., 2012) para a modelagem do crescimento animal, e de plantas e de frutos (Pires \& Calegario, 2007; Maia et al., 2009; Franco et al., 2010; Prado et al., 2013; Fernandes et al., 2014).
Como o processo de modelagem é inevitavelmente acompanhado de erros, a intenção é que eles sejam mínimos. Dessa forma, a maneira como os erros são tratados no processo é fundamentalmente importante. As estruturas normalmente utilizadas para os resíduos são de heterocedasticidade ou homocedasticidade, com erros independentes ou autorregressivos. Conjuntos de dados, no entanto, consistem de observações ordenadas no tempo, o que os torna autocorrelacionados. Box \& Jenkins (1976) propuseram uma metodologia para modelar essa correlação, a dos modelos da família ARIMA (modelos autorregressivos integrados de médias móveis). A ideia é descrever a observação em um determinado tempo como a combinação linear de valores passados da série e, também, de erros passados. Contudo, essa metodologia somente é apropriada para séries temporais estacionárias (Morettin \& Toloi, 2006).

Em grande parte dos experimentos, porém, a coleta de dados não é feita de forma regular; ou seja, as observações são feitas em intervalos de tempo 
diferentes. Na maioria desses casos, a série irregular é transformada em regular por meio de algum processo de imputação (Erdogan et al., 2005; Eckner, 2012, 2013). Esse procedimento, entretanto, pode prejudicar a qualidade do ajuste dos modelos aos dados, uma vez que fere a pressuposição da maioria das técnicas utilizadas, que consideram dados regularmente coletados.

O objetivo deste trabalho foi propor modelos que considerem estrutura irregular dos dados e avaliá-los em relação a modelos utilizados com tempos regulares.

\section{Material e Métodos}

Utilizou-se como base teórica a ideia proposta por Erdogan et al. (2005), que propõe um modelo para analisar séries irregulares. Foram considerados dados reais e simulados. Os dados reais consistiram de informações relativas ao peso corporal de animais machos não castrados - da raça Hereford, obtidos no trabalho de Emiliano (2013). Os pesos médios de 160 animais, em 16 pesagens, estão descritos na Tabela 1.

Os dados da Tabela 1 constituem uma série temporal $\mathrm{Y}_{\mathrm{t}}=\mathrm{T}_{\mathrm{t}}+\mathrm{e}_{\mathrm{t}}$, em que $\mathrm{T}_{\mathrm{t}}$ é a componente de tendência, e $e_{t}$ é a componente aleatória, estacionária, com média zero e variância constante, $e_{t} \sim \phi_{0, \sigma^{2}}$. Assim, a componente de tendência foi representada pelos modelos Logístico, Gompertz e Von Bertalanffy, com as seguintes expressões, respectivamente:

$$
\begin{gathered}
\mathrm{T}_{\mathrm{i}}=\beta_{1}\left(1+\mathrm{e}^{\beta_{2}-\beta_{3} \mathrm{x}_{\mathrm{i}}}\right)^{-1} ; \quad \mathrm{T}_{\mathrm{i}}=\beta_{1} \mathrm{e}^{\left(-\mathrm{e}^{\left(\beta_{2}-\beta_{3} \mathrm{x}_{\mathrm{i}}\right)}\right)} ; \mathrm{e} \\
\mathrm{T}_{\mathrm{i}}=\beta_{1}\left(1-\beta_{2} \mathrm{e}^{-\beta_{3} \mathrm{x}_{\mathrm{i}}}\right)^{3} ;
\end{gathered}
$$

em que o parâmetro $\beta_{1}$ representa o peso adulto máximo ou assintótico do animal, e o parâmetro $\beta_{3}$ representa a taxa de maturidade ou a velocidade do crescimento. De modo geral, não há uma interpretação prática para $\beta_{2}$ (Silveira et al., 2010).

Inicialmente, os modelos Logístico, Gompertz e Von Bertalanffy foram ajustados aos dados por meio da função GNLS do programa R (R Development Core Team, Viena, Austria). Esse procedimento foi utilizado para a obtenção dos parâmetros dos modelos, que representam a componente de tendência da série temporal $(\widehat{T})$. Por se tratar de uma função que utiliza métodos iterativos, foi necessário o uso de valores iniciais para sua implementação. Esses valores iniciais são comumente chamados de "chutes iniciais".
No presente trabalho, foi utilizado um máximo de mil e novecentos valores iniciais, em cada ajuste, para se tentar a convergência do método. Para verificar a qualidade do ajuste, foram obtidos os resíduos $e_{t}, e_{t}=Y_{t}-\widehat{T}_{t}$, além de consideradas as diferentes estruturas para os resíduos, ou seja, as estruturas regular e irregular. Os resíduos foram tratados como autorregressivos de primeira ordem, [AR(1)], em ambos os casos. Para o caso regular, o parâmetro autorregressivo foi estimado conjuntamente com os outros parâmetros. Em cada caso, os erros tiveram as seguintes expressões:

$$
e_{t}=\varphi e_{t-1}+a_{t}, e_{t}=\lambda^{\delta_{i}} e_{t-1}+a_{t}, \operatorname{com} \delta_{i}=\frac{\Delta_{i}}{\min \left(\Delta_{i}\right)},
$$

em que $\varphi$ é o parâmetro autorregressivo para o caso regular; $\lambda$ é o parâmetro autorregressivo para o caso irregular; $\delta_{\mathrm{i}}$ é uma transformação linear das distâncias reais entre duas observações consecutivas $\left(\Delta_{\mathrm{i}}\right)$; $\operatorname{emin}\left(\Delta_{\mathrm{i}}\right)$ refere-se à menor distância entre duas observações consecutivas. Essa parametrização foi utilizada para otimizar os cálculos computacionais que, no modelo original de Erdogan et al. (2005), seriam de difícil realização por causa da incapacidade computacional. Essa proposta reduz o espaço paramétrico do $\varphi$, que é de $(-1,1)$ para o intervalo $0<\lambda<1$. A minimização da soma de quadrados dos resíduos foi obtida por meio da construção de um gride para o espaço paramétrico, numa escala de 0,001 , tendo-se obtido o conjunto

Tabela 1. Valores médios dos pesos observados em diferentes idades, em 160 animais da raça Hereford, de acordo com Emiliano (2013).

\begin{tabular}{lc}
\hline Idade (dias) & Peso $(\mathrm{kg})$ \\
\hline 0 & 35,00 \\
168 & 189,97 \\
214 & 213,76 \\
243 & 243,64 \\
275 & 281,12 \\
311 & 324,39 \\
344 & 363,90 \\
377 & 404,22 \\
414 & 443,92 \\
472 & 489,51 \\
508 & 518,54 \\
542 & 541,34 \\
594 & 559,21 \\
624 & 583,81 \\
660 & 611,76 \\
692 & 639,24 \\
\hline
\end{tabular}

Pesq. agropec. bras., Brasília, v.50, n.11, p.1114-1119, nov. 2015 DOI: 10.1590/S0100-204X2015001100015 
$\{0,001 ; 0,002 ; \ldots ; 0,999\}$ como espaço paramétrico. Com o apoio computacional, percorreu-se este gride em busca do valor que minimiza a soma de quadrados, para estimar o do parâmetro $\lambda$. Este procedimento foi realizado inicialmente com dados reais de crescimento animal, obtidos em Mazzini et al. (2005) e Emiliano (2013).

Há vários métodos para se medirou avaliar a qualidade de ajuste de um modelo. No presente trabalho, foram utilizados o critério de informação de Akaike (AIC) e a soma de quadrados dos resíduos (SQR), dados por:

$$
\mathrm{AIC}=\ln (\mathrm{QME})+2(\mathrm{p}+1) \mathrm{n}, \mathrm{SQR}=\sum_{\mathrm{i}=1}^{\mathrm{n}}\left(\mathrm{y}_{\mathrm{i}}-\widehat{\mathrm{y}}_{\mathrm{i}}\right)^{2},
$$

em que QME é o quadrado médio dos resíduos; $\mathrm{p}$ é o número de parâmetros do modelo; n é o tamanho da amostra; $\mathrm{y}_{\mathrm{i}}$ é o valor observado; e $\widehat{\mathrm{y}}_{\mathrm{i}}$ é o valor ajustado.

Para os dados simulados, foram utilizados os valores paramétricos obtidos do ajuste dos modelos de crescimento com os dados reais. As estimativas dos parâmetros do modelo mais bem ajustado foram utilizadas na simulação de dados, para cada um dos modelos. Para cada um deles, foram simulados pesos de animais do $1^{\circ}$ ao $730^{\circ}$ dia após o nascimento $(n=730)$. Em cada simulação, os erros foram gerados como autorregressivos de primeira ordem. O respectivo modelo foi ajustado a cada simulação; por exemplo: para a simulação feita com o modelo Logístico, a curva logística foi ajustada, com a consideração das diferentes estruturas de erros (regular e irregular). Em seguida, amostras aleatórias de tamanho 100 foram retiradas, sucessivamente, das simulações, e o modelo foi reajustado para as observações restantes. A cada retirada de uma amostra, os modelos foram novamente ajustados e comparados. Portanto, foram feitos ajustes com diferentes tamanhos amostrais, $\mathrm{n}=730,630,530$, $430,330,230,130$ e 30, para verificar a eficiência do método considerado no presente trabalho.

\section{Resultados e Discussão}

Os valores estimados dos parâmetros dos modelos Gompertz, Logístico e Von Bertalanffy, bem como o valor de AIC em cada caso, obtidos com o ajuste aos dados reais que minimizaram as somas de quadrados dos resíduos, quando consideradas as estruturas regular e irregular destes, estão descritos na Tabela 2.
Ao se considerar a estrutura irregular dos resíduos, o ajuste dos modelos melhorou; no caso do modelo Von Bertalanffy, a soma de quadrados de resíduos chegou a diminuir 18,5\%. As estimativas dos parâmetros autorregressivos também mudaram, quando essa estrutura foi considerada; no caso do modelo Logístico, a estimativa chegou a mudar de sinal (Tabela 2). Em todos os ajustes, o valor de AIC foi menor para os modelos com erros irregulares, tendo seguido a mesma tendência das somas de quadrados e indicado que há melhoria no ajuste quando essa estrutura é considerada. Para cada um dos modelos, foram gerados 100 conjuntos de dados de crescimento com as estimativas obtidas para os parâmetros dos modelos que melhor se ajustaram aos dados.

Os valores médios dos parâmetros para os dados simulados estão apresentados nas Tabelas 3, 4 e 5 . Os diferentes tamanhos amostrais nessas tabelas representam o grau de irregularidade presente em situações reais ou a maior disponibilidade de informação sobre o evento estudado. Verificou-se que as estimativas dos parâmetros foram mais precisas à medida que mais informação sobre o fenômeno estava disponível. Erdogan et al. (2005) também relataram decréscimo na precisão da estimativa do parâmetro conforme a amostra de dados diminuía. As estimativas médias dos parâmetros aproximaram-se bem das estimativas reais, o que também foi observado por

Tabela 2. Valores estimados dos parâmetros e do critério de informação de Akaike (AIC) obtidos com o ajuste dos modelos aos dados reais, ao se considerar as estruturas regular e irregular dos dados, e as somas de quadrados dos resíduos em ambos os casos, para os diferentes modelos avaliados.

\begin{tabular}{|c|c|c|c|c|c|c|}
\hline \multirow[t]{2}{*}{ Parâmetro $^{(1)}$} & \multicolumn{2}{|c|}{ Logístico } & \multicolumn{2}{|c|}{ Gompertz } & \multicolumn{2}{|c|}{ Von Bertalanffy } \\
\hline & Regular & Irregular & Regular & Irregular & Regular & Irregular \\
\hline$\beta_{1}$ & 660,786 & 660,854 & 746,165 & 745,448 & 827,891 & 819,245 \\
\hline$\beta_{2}$ & 9,2077 & 9,2703 & 1,0686 & 1,0691 & 0,6615 & 0,6681 \\
\hline$\underline{\beta_{3}}$ & 0,0070 & 0,0070 & 0,0041 & 0,0041 & 0,0030 & 0,0030 \\
\hline $\bar{\varphi}$ & $-0,1446$ & - & 0,1428 & - & 0,4179 & - \\
\hline SQR & 2.339 & - & 1.194 & - & 1.322 & - \\
\hline$\lambda$ & - & 0,4477 & - & 0,3632 & - & 0,4695 \\
\hline$\underline{\text { SQIR }}$ & - & 2.156 & - & 1.090 & - & 1.076 \\
\hline & \multicolumn{6}{|c|}{ Valores de AIC } \\
\hline E. regulares & \multicolumn{2}{|c|}{5,8956} & \multicolumn{2}{|c|}{5,2257} & \multicolumn{2}{|c|}{5,3276} \\
\hline E. irregulares & \multicolumn{2}{|c|}{5,8163} & \multicolumn{2}{|c|}{5,1348} & \multicolumn{2}{|c|}{5,1215} \\
\hline
\end{tabular}

${ }^{(1)} \varphi$, parâmetro autorregressivo para o caso regular; SQR, soma de mínimos quadrados dos resíduos para a estrutura regular; SQIR, soma de mínimos quadrados para a estrutura irregular; $\lambda$, parâmetro autorregressivo para $o$ caso irregular; e E., erros. 
Erdogan et al. (2005). A maior diferença foi notada na estimativa média do parâmetro autorregressivo, quando a estrutura irregular foi considerada. À medida que as amostras são retiradas, as estimativas desse parâmetro distanciam-se da estimativa real; mas, em todos os casos, sua estimativa média é mais próxima da estimativa real do que quando a irregularidade nos dados não é considerada.

Tabela 3. Valores médios estimados para os parâmetros do modelo Logístico, para os dados $\operatorname{simulados}^{(1)}$.

\begin{tabular}{|c|c|c|c|c|c|c|c|c|}
\hline \multirow[t]{2}{*}{$\mathrm{n}$} & \multicolumn{3}{|c|}{ Estrutura regular } & \multirow[t]{2}{*}{$\varphi$} & \multicolumn{3}{|c|}{ Estrutura irregular } & \multirow[t]{2}{*}{$\lambda$} \\
\hline & $\beta_{1}$ & $\beta_{2}$ & $\beta_{3}$ & & $\beta_{1}$ & $\beta_{2}$ & $\beta_{3}$ & \\
\hline 30 & 660,695 & 9,3066 & 7,03389E-03 & $-0,0791$ & 660,657 & 9,3078 & 7,03482E-03 & 0,3191 \\
\hline 130 & 660,943 & 9,2805 & 7,01980E-03 & 0,0957 & 660,932 & 9,2808 & 7,02003E-03 & 0,3923 \\
\hline 230 & 660,875 & 9,2838 & 7,02365E-03 & 0,1905 & 660,871 & 9,2835 & 7,02362E-03 & 0,4323 \\
\hline 330 & 658,000 & 9,1916 & 7,47298E-03 & 0,2731 & 660,894 & 9,2840 & 7,02471E-03 & 0,4390 \\
\hline 430 & 657,773 & 9,1913 & 7,88200E-03 & 0,3244 & 660,724 & 9,2817 & $7,02626 \mathrm{E}-03$ & 0,4397 \\
\hline 530 & 660,593 & 9,2837 & $7,02819 \mathrm{E}-03$ & 0,3610 & 660,589 & 9,2835 & $7,02817 \mathrm{E}-03$ & 0,4384 \\
\hline 630 & 657,741 & 9,1868 & $8,26801 \mathrm{E}-03$ & 0,4091 & 660,729 & 9,2789 & 7,02492E-03 & 0,4406 \\
\hline 730 & 651,488 & 9,0046 & $2,22270 \mathrm{E}-02$ & 0,4611 & 660,741 & 9,2790 & $7,02442 \mathrm{E}-03$ & 0,4439 \\
\hline
\end{tabular}

(1)Valores reais utilizados na simulação: $\beta_{1}, 660,854 ; \beta_{2}, 9,2703 ; \beta_{3}, 7,02 \mathrm{E}-3$; e $\lambda, 0,4477$. $\varphi$, parâmetro autorregressivo para o caso regular; e $\lambda$, parâmetro autorregressivo para o caso irregular.

Tabela 4. Valores médios estimados ${ }^{(1)}$ para os parâmetros do modelo Gompertz, para os dados simulados.

\begin{tabular}{|c|c|c|c|c|c|c|c|c|}
\hline \multirow[t]{2}{*}{$\mathrm{n}$} & \multicolumn{3}{|c|}{ Estrutura regular } & \multirow[t]{2}{*}{$\varphi$} & \multicolumn{3}{|c|}{ Estrutura irregular } & \multirow[t]{2}{*}{$\lambda$} \\
\hline & $\beta_{1}$ & $\beta_{2}$ & $\beta_{3}$ & & $\beta_{1}$ & $\beta_{2}$ & $\beta_{3}$ & \\
\hline$\overline{30}$ & 743,268 & 1,0705 & $4,09787 \mathrm{E}-03$ & $-0,0952$ & 743,351 & 1,0703 & 4,09669E-03 & 0,3294 \\
\hline 130 & 745,144 & 1,0693 & $4,07554 \mathrm{E}-03$ & 0,0603 & 745,122 & 1,0693 & $4,07570 \mathrm{E}-03$ & 0,3247 \\
\hline 230 & 744,646 & 1,0694 & $4,07875 \mathrm{E}-03$ & 0,1301 & 744,651 & 1,0694 & 4,07876E-03 & 0,3306 \\
\hline 330 & 744,637 & 1,0693 & $4,07800 \mathrm{E}-03$ & 0,1854 & 744,650 & 1,0693 & 4,07793E-03 & 0,3339 \\
\hline 430 & 744,785 & 1,0692 & $4,07680 \mathrm{E}-03$ & 0,2415 & 744,796 & 1,0692 & 4,07673E-03 & 0,3461 \\
\hline 530 & 744,524 & 1,0696 & $4,07944 \mathrm{E}-03$ & 0,2829 & 744,538 & 1,0696 & 4,07936E-03 & 0,3488 \\
\hline 630 & 744,610 & 1,0692 & $4,07755 \mathrm{E}-03$ & 0,3194 & 744,619 & 1,0692 & $4,07751 \mathrm{E}-03$ & 0,3511 \\
\hline 730 & 744,761 & 1,0689 & $4,07580 \mathrm{E}-03$ & 0,3520 & 744,772 & 1,0689 & 4,07574E-03 & 0,3520 \\
\hline
\end{tabular}

${ }^{(1)}$ Valores reais utilizados na simulação: $\beta_{1}, 745,4478 ; \beta_{2}, 1,0691 ; \beta_{3}, 4,07 \mathrm{E}-3$; e $\lambda, 0,3632$. $\varphi$, parâmetro autorregressivo para o caso regular; e $\lambda$, parâmetro autorregressivo para o caso irregular.

Tabela 5. Valores médios estimados para os parâmetros do modelo Von Bertalanffy, para os dados simulados ${ }^{(1)}$.

\begin{tabular}{|c|c|c|c|c|c|c|c|c|}
\hline \multirow[t]{2}{*}{$\mathrm{n}$} & \multicolumn{3}{|c|}{ Estrutura regular } & \multirow[t]{2}{*}{$\varphi$} & \multicolumn{3}{|c|}{ Estrutura irregular } & \multirow[t]{2}{*}{$\lambda$} \\
\hline & $\beta_{1}$ & $\beta_{2}$ & $\beta_{3}$ & & $\beta_{1}$ & $\beta_{2}$ & $\beta_{3}$ & \\
\hline 30 & 819,738 & 0,6690 & $3,01043 \mathrm{E}-03$ & $-0,0847$ & 819,680 & 0,6690 & $3,01047 \mathrm{E}-03$ & 0,3454 \\
\hline 130 & 819,087 & 0,6762 & $3,01470 \mathrm{E}-03$ & 0,1222 & 819,477 & 0,6683 & 3,00892E-03 & 0,4235 \\
\hline 230 & 819,226 & 0,6686 & $3,01205 \mathrm{E}-03$ & 0,1959 & 819,240 & 0,6686 & $3,01200 \mathrm{E}-03$ & 0,4438 \\
\hline 330 & 819,465 & 0,6684 & 3,00961E-03 & 0,2811 & 819,478 & 0,6684 & $3,00956 \mathrm{E}-03$ & 0,4591 \\
\hline 430 & 820,007 & 0,6682 & $3,00642 \mathrm{E}-03$ & 0,3227 & 820,009 & 0,6682 & $3,00645 \mathrm{E}-03$ & 0,4482 \\
\hline 530 & 819,967 & 0,6682 & $3,00665 \mathrm{E}-03$ & 0,3779 & 819,996 & 0,6682 & $3,00652 \mathrm{E}-03$ & 0,4552 \\
\hline 630 & 819,929 & 0,6683 & $3,00710 \mathrm{E}-03$ & 0,4182 & 819,942 & 0,6683 & $3,00703 \mathrm{E}-03$ & 0,4559 \\
\hline 730 & 819,648 & 0,6685 & $3,00933 \mathrm{E}-03$ & 0,4598 & 819,671 & 0,6685 & $3,00925 \mathrm{E}-03$ & 0,4597 \\
\hline
\end{tabular}

(1)Valores reais utilizados na simulação: $\beta_{1}, 819,245 ; \beta_{2}, 0,6681 ; \beta_{3}, 0,00301 ;$ e $\lambda, 0,4695$. $\varphi$, parâmetro autorregressivo para o caso regular; e $\lambda$, parâmetro autorregressivo para o caso irregular. 
Eckner $(2012,2013)$ afirma que transformar séries irregulares em séries regulares pode causar inúmeros vieses, o que de fato ficou comprovado no resultado anterior, em que, ao se levar em consideração a estrutura original dos dados, foram obtidas melhores estimativas. No caso de $n=30$, a estimativa média do parâmetro autorregressivo regular, para todos os modelos, distanciou-se a ponto de ter havido uma mudança de sinal, o que indica que, com menor disponibilidade de dados, a estrutura real ajuda a obter estimativas mais próximas do valor real.

A cada ajuste, os modelos regular e irregular foram comparados. A Tabela 6 apresenta o número de vezes em que o modelo irregular foi superior ao modelo regular, nas 100 simulações realizadas. A avaliação foi feita com base na soma de quadrados de resíduos e nos valores dos AIC's.

Constatou-se que, em grande parte dos casos, a observância da irregularidade nos dados diminuiu os valores de AIC, o que gerou melhores ajustes (Tabela 6). Para os valores de $\mathrm{n}=30$, em 92 das 100 simulações feitas para o modelo Logístico, a estrutura irregular proporcionou melhor ajuste; no caso dos modelos Gompertz e Von Bertalanffy, esse resultado foi 90. Quando $\mathrm{n}=130$, para o modelo Logístico, 98 das 100 simulações ficaram mais bem ajustadas quando se considerou a estrutura irregular; para o modelo Gompertz, 95, e, para o Von Bertalanffy, 99. Em todos os outros casos, a observância da irregularidade dos resíduos proporcionou melhor ajuste.

Segundo Erdogan et al. (2005), é comum que se ignore, na prática, as irregularidades, e que se trate as séries como regulares, como nos trabalhos de Mazzini et al. (2005), McManus et al. (2010), Mansano et al. (2012) e Silva et al. (2011). Portanto, se a estrutura original dos dados for considerada, melhores ajustes podem ser obtidos nestes trabalhos.

Tabela 6. Número de vezes em que o modelo irregular superou o modelo regular, em 100 simulações.

\begin{tabular}{lccc}
\hline $\mathrm{n}$ & Logístico & Gompertz & Von Bertalanffy \\
\hline 30 & 92 & 90 & 90 \\
130 & 98 & 95 & 99 \\
230 & 100 & 100 & 100 \\
330 & 100 & 100 & 100 \\
430 & 100 & 100 & 100 \\
530 & 100 & 100 & 98 \\
630 & 100 & 100 & 96 \\
730 & 100 & 100 & 93 \\
\hline
\end{tabular}

Pesq. agropec. bras., Brasília, v.50, n.11, p.1114-1119, nov. 2015 DOI: 10.1590/S0100-204X2015001100015

\section{Conclusões}

1. A estrutura irregular dos resíduos leva a menores valores do critério de informação de Akaike e a menores somas de quadrados dos resíduos.

2. O modelo Von Bertalanffy, para dados reais, é o que proporciona a menor soma de quadrados dos resíduos.

3. Independentemente do tamanho amostral, as estimativas médias evidenciam a eficiência do método proposto no presente trabalho, na presença de irregularidades, que apresentou valores mais próximos dos reais.

\section{Agradecimentos}

À Coordenação de Aperfeiçoamento de Pessoal de Nível Superior (Capes) e à Fundação de Amparo à Pesquisa do Estado de Minas Gerais (Fapemig), pela concessão de bolsas.

\section{Referências}

BOX, G.E.P.; JENKINS, G.M. Time series analysis: forecasting and control. San Francisco: Holden-Day, 1976. 575p.

COSTA, A.C.; REIS NETO, R.V.; FREITAS, R.T.F.; FREATO, T.A.; LAGO, A.A.; SANTOS, V.B. Avaliação do crescimento de tilápias de diferentes linhagens através de modelos não lineares. Archivos de Zootecnia, v.58, p.561-564, 2009. Suplemento.

ECKNER, A. A framework for the analysis of unevenly spaced time series data. 2013. Available at: $<$ http://eckner.com/papers/ unevenly_spaced time series analysis.pdf $>$. Accessed on: 22 abr. 2013.

ECKNER, A. A note on trend and seasonality estimation for unevenly-spaced time series. 2012. Available at: <http://www. eckner.com/papers/trend_and_seasonality.pdf $>$. Accessed on: 22 abr. 2013

EMILIANO, P.C. Critérios de informação: como eles se comportam em diferentes modelos? 2013. 179p. Tese (Doutorado) - Universidade Federal de Lavras, Lavras.

ERDOGAN, E.; MA, S.; BEYGELZIMER, A.; RISH, I. Statistical models for unequally spaced time series. In: SIAM INTERNATIONAL CONFERENCE ON DATA MINING, 2005, Auckland. Proceedings. Auckland: University of Auckland, 2005. p.626-630. DOI: 10.1137/1.9781611972757.74.

FERNANDES, T.J.; PEREIRA, A.A.; MUNIZ, J.A.; SAVIAN, T.V. Seleção de modelos não lineares para a descrição das curvas de crescimento do fruto do cafeeiro. Coffee Science, v.9, p.207-215, 2014.

FRANCO, L.R.L.; SILVA, J.F.; MAIA, V.M.; LOPES, P.S.; AMORIM, I. de J.F.; MIZOBUTSI, E.H. Pegamento e crescimento inicial de mudas de jabuticabeiras 'Açu' e 'Sabará' submetidas a 
dois tipos de enxertia. Revista Ceres, v.57, p.535-538, 2010. DOI: 10.1590/S0034-737X2010000400016.

GOMIERO, J.S.G.; FREITAS, R.T.F. de; SANTOS, V.B. dos; SILVA, F.F. da; RODRIGUES, P.B.; LOGATO, P.V.R. Curvas de crescimento morfométrico de piracanjuba (Brycon orbignyanus). Ciência e Agrotecnologia, v.33, p.882-889, 2009. DOI: 10.1590/ S1413-70542009000300031.

GONÇALVES, T. de M.; DIAS, M.A.D.; AZEVEDO JUNIOR, J.; RODRIGUEZ, M.A.P.; TIMPANI, V.D.; OLIVEIRA, A.I.G. de. Curvas de crescimento de fêmeas da raça Nelore e seus cruzamentos. Ciência e Agrotecnologia, v.35, p.582-590, 2011. DOI: $10.1590 / \mathrm{S} 1413-70542011000300021$.

MAIA, E.; SIQUEIRA, D.L. de; SILVA, F.F. e; PETERNELli, L.A.; SALOMÃO, L.C.C. Método de comparação de modelos de regressão não-lineares em bananeiras. Ciência Rural, v.39, p.1380-1386, 2009. DOI: 10.1590/S0103-84782009000500012.

MANSANO, C.F.M.; STÉFANI, M.V. de; PEREIRA, M.M.; MACENTE, B.I. Non-linear growth models for bullfrog tadpoles. Ciência e Agrotecnologia, v.36, p.454-462, 2012. DOI: 10.1590/ S1413-70542012000400010.

MAZUCHELI, J.; SOUZA, R.M. de; PHILIPPSEN, A.S. Modelo de crescimento de Gompertz na presença de erros normais heterocedásticos: um estudo de caso. Revista Brasileira de Biometria, v.29, p.91-101, 2011.

MAZZINI, A.R. de A.; MUNIZ, J.A.; SILVA, F.F. e; AQUINO, L.H. de. Curva de crescimento de novilhos Hereford: heterocedasticidade e resíduos autorregressivos. Ciência Rural, v.35, p.422-427, 2005. DOI: 10.1590/S0103-84782005000200028.
McMANUS, C.M.; LOUVANDINI, H.; CAMPOS, V.A.L. Non linear growth curves for weight and height in four genetic groups of horses. Ciência Animal Brasileira, v.11, p.80-89, 2010.

MORETTIN, P.A.; TOLOI, C.M.C. Análise de séries temporais. 2.ed. São Paulo: E. Blucher, 2006. 560p.

PIRES, L.M.; CALEGARIO, N. Ajustes de modelos estocásticos lineares e não-lineares para a descrição do perfil longitudinal de árvores. Revista Árvore, v.31, p.845-852, 2007. DOI: 10.1590/ S0100-67622007000500008.

PRADO, T.K.L. do; SAVIAN, T.V.; MUNIZ, J.A. Ajuste dos modelos Gompertz e Logístico aos dados de crescimento de frutos de coqueiro anão verde. Ciência Rural, v.43, p.803-809, 2013. DOI: $10.1590 / \mathrm{S} 0103-84782013005000044$.

SILVA, F. de L.; ALENCAR, M.M. de; FREITAS, A.R. de; PACKER, I.U.; MOURÃO, G.B. Curvas de crescimento em vacas de corte de diferentes tipos biológicos. Pesquisa Agropecuária Brasileira, v.46, p.262-271, 2011. DOI: 10.1590/ S0100-204X2011000300006.

SILVEIRA, F.G. da; SILVA, F.F. e; CARNEIRO, P.L.S.; MALHADO, C.H.M. Classificação multivariada de modelos de crescimento para grupos genéticos de ovinos de corte. Revista Brasileira de Saúde e Produção Animal, v.13, p.62-73, 2012. DOI: 10.1590/S1519-99402012000100006.

SOUZA, L. de A.; CAIRES, D.N.; CARNEIRO, P.L.S.; MALHADO, C.H.M.; MARTINS FILHO, R. Curvas de crescimento em bovinos da raça Indubrasil criados no estado do Sergipe. Revista Ciência Agronômica, v.41, p.671-676, 2010. DOI: $10.1590 /$ S1806-66902010000400022.

Recebido em 19 de novembro de 2014 e aprovado em 31 de agosto de 2015 